\section{TELEPRESENCE CONFOCAL MICROSCOPY}

Youngblom, J. H., Wilkinson, J., and Youngblom, J.J.

California State University-Stanislaus

The advent of the Internet has allowed the development of remote access capabilities to a growing variety of microscopy systems. The Materials MicroCharacterization Collaboratory, for example, has developed an impressive facility that provides remote access to a number of highly sophisticated microscopy and microanalysis instruments. While certain types of microscopes, such as scanning electron microscopes, transmission electron microscopes, scanning probe microscopes, and others have already been established for telepresence microscopy, no one has yet reported on the development of similar capabilities for the confocal laser scanning microscope.

At California State University-Stanislaus, home of the CSUPERB (California State University Program for Education and Research in Biotechnology) Confocal Microscope Core Facility, we have established a remote access confocal laser scanning microscope facility that allows users with virtually any type of computer platform to connect to our system. Our Leica TCS NT confocal system, with an interchangeable upright (DMRXE) and inverted microscope (DMIRBE) set up, is accessible to any authorized user via the Internet by using a FREE software program called VNC (Virtual Network Computing). Although there are other commercially available software programs available for remote connectivity, such as PC Anywhere and Timbuktu, they have some significant disadvantages. Two of the main problems involve the monetary cost associated with the need to have the software program installed at both ends of the connection, and secondly each program is computer platform dependent. Largely for these reasons, we have chosen to use the VNC software program for all our remote connections. It is, in essence, a remote display system which allows users to view a computing 'desktop' environment not only on the machine where it is running, but from anywhere on the Internet and using virtually any computer platform. These features allow our confocal system to be available to a far wider audience and user base. Information and instructions regarding scheduling and remote access to our confocal microscope system are available at our web site, http://science.csustan.edu/confocal.

A technical assistant is on hand to initially set the specimen under the microscope and provide the initial plane of focus. Once connectivity is established, the remote user is able to control virtually all the functions to conduct real time image analysis and quantitative assessments of the specimen. These functions include: filter selection, zoom, focus, adjustment of pinhole size, PMT intensity for each of the three separate channels, $x-y$ stage movement, parameters for optical sectioning, and laser attenuation using the Acousto Optical Tunable Filter. The critical component of the system that allows this remote control is the electronic "panel box" that drives most of these functions. A motorized stage mounted on the upright microscope allows users to scan their entire specimen by moving the stage electronically. The Winpos software program allows remote users to move the stage anywhere within a confined area of the slide that is adjusted by the staff at the core facility. Users are also able to control the velocity and acceleration rate of the movement. Additionally, the program provides absolute $\mathrm{x}$ and $\mathrm{y}$ coordinate values for each location viewed on the slide, so that finding a particular site of interest at a

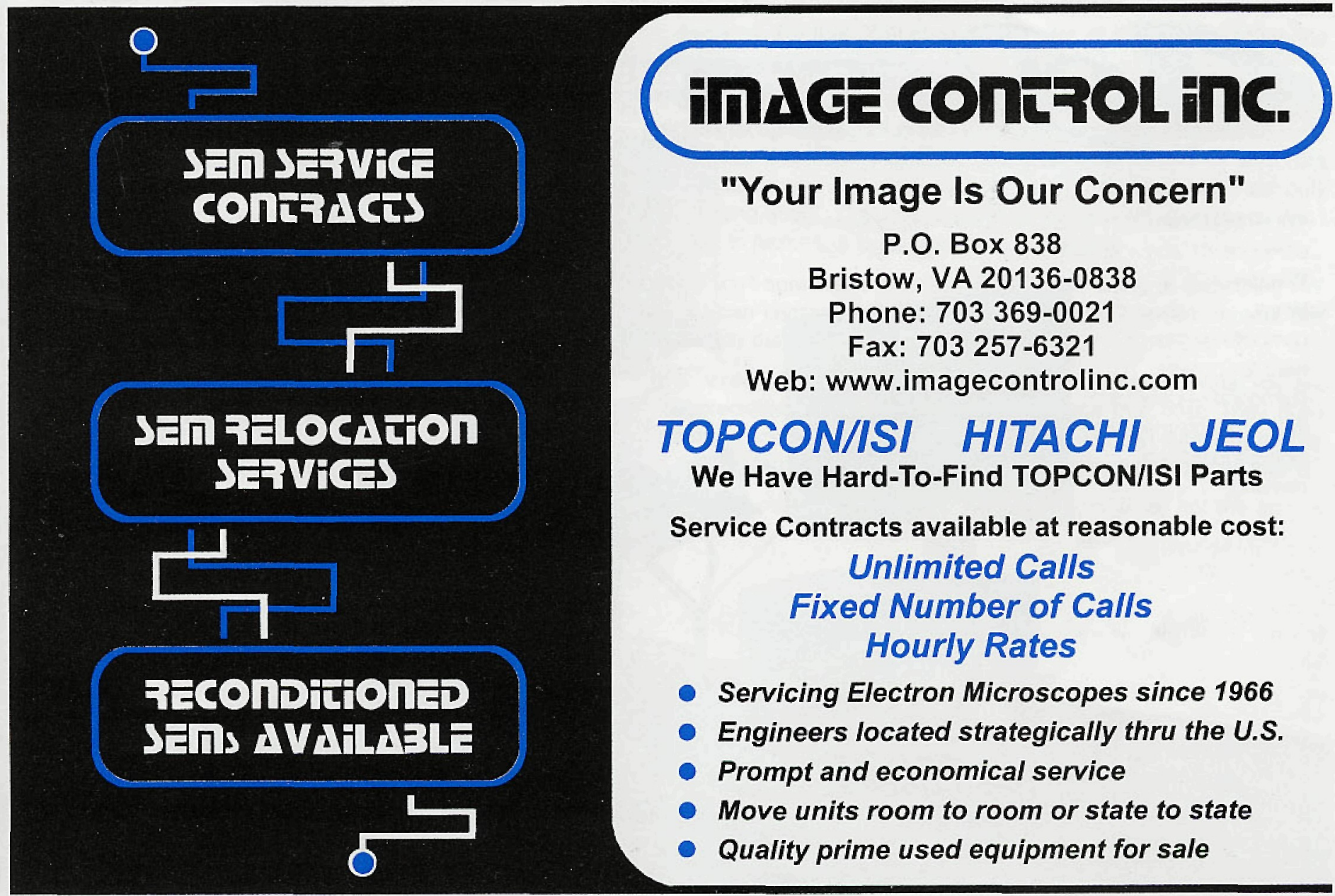




\section{later time is assured}

Quantitative analysis of the scanned images can be conducted on-line and the data saved by screen capture or other methods. Two-dimensional stacks can also be used to generate psuedo 3-D images on-line using a Leica program available on the confocal computer, or reconstructed into 3-D images using the Voxblast software (Vaytek, Inc.) available on-line on a separate workstation at the Confocal Core Facility. Freeware programs, such as $\mathrm{NIH}$ image or Scionlmage, may also be downloaded directly from the internet for 3D reconstruction by the user on his or her own computer.

Images acquired during a session are directly saved into a folder located on the confocal computer desktop. This folder is linked to the CSUPERB Confocal web site and remote users are able to download their saved images directly onto their own computer at the end of their session by going to the web site. The images are subsequently deleted from the folder on the confocal computer desktop at the end of the session. Users can request a backup zip disc made and be retained at the confocal core facility. Once a user is done with a session, she/he can turn off the VNC program on the confocal computer and thus terminate the session.

Communication between the remote user and staff at the Confocal Core Facility can be maintained throughout the session by using a free internet software program called NetMeeting. This program allows real time auditory contact between the two sites comparable to a regular telephone connection, but without the cost. One- or two-way visual contact between the sites is also an option. The program provides text chat, a white board, and file transfer features that support a comprehensive environment for real-time conferencing and collaboration. One limitation to this program is that it only exists for PC computers at the present time.

We have strived to develop a prototype remote access confocal microscope system that is relatively inexpensive, simple to set up and maintain, and is highly efficient. We have used free software off the Internet whenever possible, or have developed our own software solutions that we will freely share with the public. The intent of this approach is to minimize the cost barriers that may discourage centers from considering remote access development of their own instruments, as well as to open up the spectrum of remote users that can access the instruments. This technology is having its greatest impact on education where students and faculty at multiple institutions are able to simultaneously connect to the instrument for viewing real time specimen demonstrations, collaboration on research projects, and sharing of specimen resources among a diverse academic community.

1. This work was funded in part by the National Science Foundation's Division of Undergraduate Education through grant DUE \# 9651379

Microsc. Microanal. 6 (Suppl 2: Proceedings): 1164-1165 (2000); reprinted with the permission of the publisher, Springer-Verlag, NY.

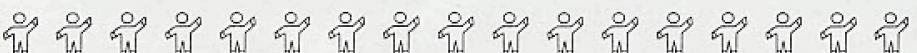

The microscope with its accessories is by far the least

of understood, the most inefficiently operated, and the most

qu abused of all the laboratory instruments.

". . . Shillaber (Author of the "classic" LM text, circa 1950)

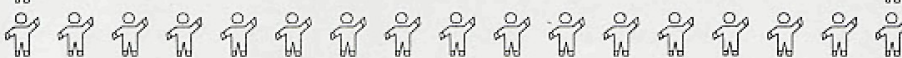

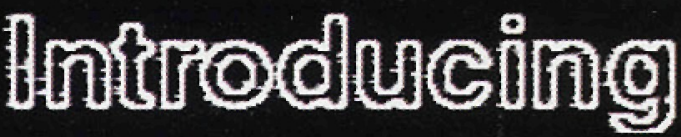

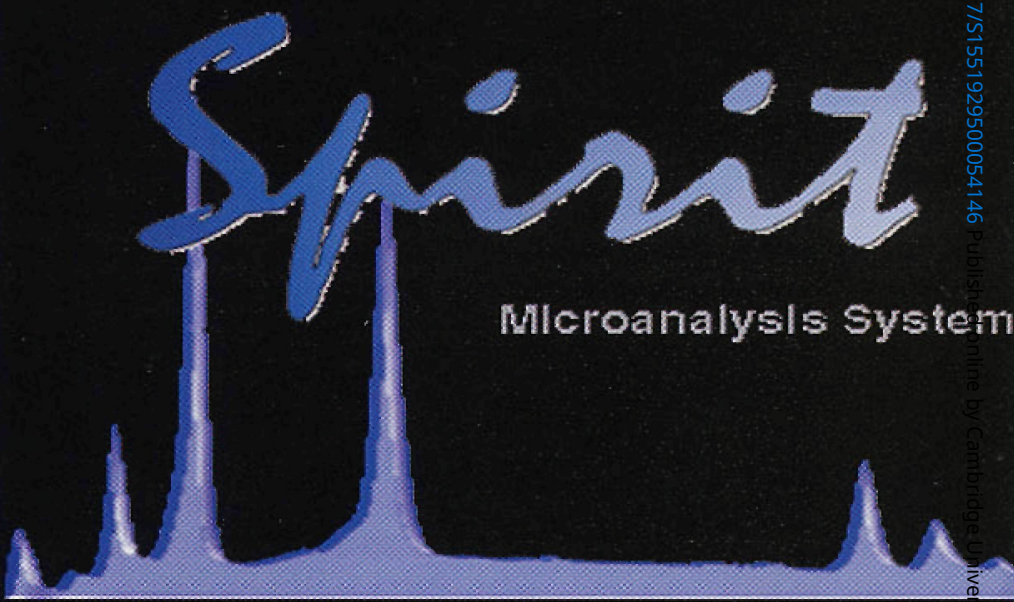

PGT Introduces a Hi-Spirited, Hi-Speed, EDS system for Electron Microscopy. . .

- If complete sample data is what you are after, you need Spirit!

- If time is of the essence, you need Spirit.

- If you are familiar with the Microsoft look \& feel, you can run Spirit!

Take a look at Spirit - the fastest mapping available using PGT's award winning Position-Tagged Spectrometry. See how fast it really is:

\section{www.pgt.com/spirit.htm}
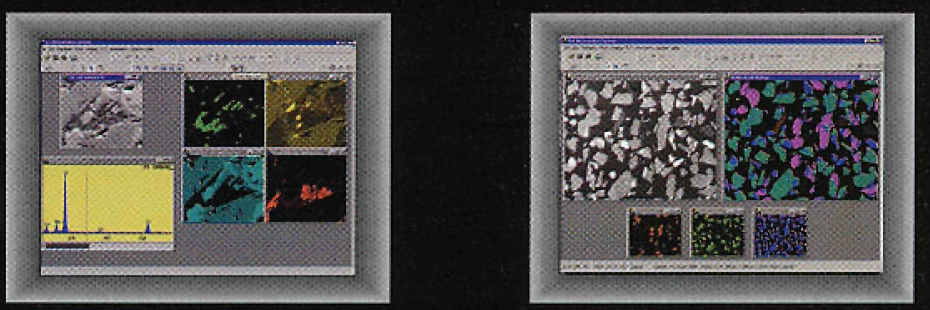

Princeton Gamma-Tech, Inc. C/N 863 Princeton, NJ 08542-0863 Tel: (609) 924-7310 Toll Free: 800-229-7484 Fax: (609) 924-1729 Website: www.pgt.com E-mail: sales @epgt.com 